\title{
Evaluation of dentin hypersensitivity treatment with glass ionomer cements: A randomized clinical trial
}

\section{Marina de Matos MADRUGA ${ }^{(a)}$ Adriana Fernandes da SILVA(b) Wellington Luiz de Oliveira da ROSA(a) Evandro PIVA(b) Rafael Guerra LUND(b)}

(a) Universidade Federal de Pelotas - UFPEL, School of Dentistry, Program of Post-Graduate in Dentistry, Pelotas, RS, Brazil;

(b) Universidade Federal de Pelotas - UFPEL, School of Dentistry, Department of Restorative Dentistry, Pelotas, RS, Brazil.
Declaration of Interests: The authors certify that they have no commercial or associative interest that represents a conflict of interest in connection with the manuscript.

\section{Corresponding Author:}

Rafael Guerra Lund

E-mail: rafael.lund@gmail.com

DOI: 10.1590/1807-3107BOR-2017.vol31.0003

Submitted: Apr 17, 2016

Accepted for publication: Oct 21, 2016

Last revision: Nov 21, 2016

\begin{abstract}
A randomized, double-blind, split-mouth clinical trial was performed compared the desensitizing efficacy of the resin-modified glass ionomer cement (GIC) ClinproTM XT (3M ESPE, Minnesota, USA) and the conventional GIC Vidrion R (SS White, Gloucester, UK) in a 6-month follow-up. Subjects were required to have at least two teeth with dentin hypersensitivity. Teeth were divided at random into 2 groups, one group received Clinpro XT and the other conventional GIC Vidrion R. Treatments were assessed by tactile and air blast tests using Visual Analogue Scale (VAS) at baseline, after 20 minutes, and at 7, 15, 21, 30, 90 and 180 days post-treatment. Twenty subjects (152 teeth) were included. Both tests (tactile and air blast) showed a significant reduction of dentin hypersensitivity immediately after the application of Vidrion R and Clinpro XT (20 min). VAS scores obtained along the 6-month follow-up were statistically lower when compared to initial rates $(p<0.05)$. Both GIC were able to reduce dentin hypersensitivity up to 6-month post-treatment period without statistically significant differences among them $(p>0.05)$. Both cements provided satisfactory results in long-term dental sensitivity reduction.
\end{abstract}

Keywords: Dentin Sensitivity; Dentin Desensitizing Agents; Glass Ionomer Cements; Randomized Controlled Trial.

\section{Introduction}

Dentin hypersensitivity $(\mathrm{DH})$ is characterized by a painful reaction due to the exposure of dentin to chemical, thermal, tactile or osmotic non-harmful stimuli. According to the Hydrodynamic Theory, fluid movement in the dentinal tubules excites mechanoreceptors in the periphery of the pulp and triggers pain. ${ }^{1}$ Consequently, DH treatment consists of sealing the dentinal tubules or depolarizing the pulp's nerve fibers. ${ }^{1}$ The physical mechanism of the process is achieved by the application of dentin desensitizing agents such as dentin bonding agents, composite resins, glass ionomer cements (GICs) and varnishes. ${ }^{2}$ Although extensive research on dentin hypersensitive treatments has been conducted, no single treatment is currently accepted by all. ${ }^{3,4,5}$

GICs have become a popular restorative material due to their chemical adhesion and ability to release fluoride. ${ }^{2}$ Further, GICs have shown extended satisfactory results over time, especially in the treatment of 
non-carious cervical lesions. ${ }^{6}$ The pattern of fluoride release from GIC comprises a high initial release rate followed by rapid reduction. ${ }^{7}$ However, sensitivity to moisture contamination interferes with the initial setting of self-cured GIC. In addition, conventional GICs are relatively unaesthetic and have poorer mechanical properties than resin composites, ${ }^{8}$ which might impair the long-term treatment of $\mathrm{DH}$. In fact, resin-modified glass ionomer cements have been developed to overcome the disadvantages of conventional GIC. They are more tolerant to moisture and do not require drying of the tooth surface. ${ }^{9}$ The recently available Clinpro ${ }^{\mathrm{TM}}$ XT (3M ESPE, Minnesota, USA) is a new paste-liquid that may be used as a site-specific, light-cured desensitizing agent. ${ }^{10,11,12,13}$ Ion exchange with dental substrate inherent to GICs enhanced the development of these materials to obtain high fluoride release. ${ }^{14}$ Since GICs may also release therapeutic doses of fluoride over time, the activity is an important feature to obstruct tubules in $\mathrm{DH}$ treatment. ${ }^{11,13,15,16}$ However, there is no consensus in the literature regarding to the clinical relevance of released fluorides by these materials. ${ }^{17}$

The rapid reduction of $\mathrm{DH}$ and the long-term duration of these desensitizing effects are critical. Previous studies reported that the effects of several desensitizing agents were not permanent because they did not properly adhere to the dentin surface. ${ }^{6,8,13}$ However, a limited amount of data on the efficacy of desensitizers is available in the literature, especially for long-term assessments of at least 6-months follow-up. 2,4,18,19 Therefore, current randomized, split-mouth clinical trial evaluates and compares the desensitizing efficacy of Clinpro XT and conventional GIC (Vidrion R) in a 6-month follow-up. The hypothesis tested is that resin-modified GIC (Clinpro XT) would be more effective than conventional GIC (Vidrion R) in controlling dentin hypersensitivity up to a 6-month post-treatment period.

\section{Methodology}

Methodology followed guidelines for clinical trials recommended by Standard CONSORT (Consolidated Standards of Reporting Trials). ${ }^{2}$ Research protocol was approved by the local Ethics Committee (IRB approval \# 73/12).

\section{Study design}

Current randomized, double-blind (subjects and examiner) clinical trial was conducted at a School of Dentistry and it was registered at ClinicalTrials.gov (NCT02378129). All subjects were informed of the nature of the experiment. According to a previous study, ${ }^{20}$ the expected baseline mean tactile score was $6.43 \pm 2.09$ for a resin-modified glass ionomer cement. The hypothesis consisted of a mean change (from baseline to finish) of $5.66 \pm 1.39$ for the best material and $4.06 \pm 1.53$ for the worst one. Using an unpaired $t$ test and assuming an error of 0.05 and an expected withdrawal/dropout rate of $20 \%$, a minimum of 14 participants were requested to achieve $80 \%$ statistical efficiency. The plan was to conservatively enroll at least 18 subjects.

The researchers registered the detailed medical and dental history of each subject to rule out participants that did not fit the inclusion criteria. An oral and voluntary written informed consent was obtained from the participants prior to start of the study. Subjects who met all inclusion criteria signed the consent form before submitting to the trial. Inclusion criteria comprised male and female subjects between 18 and 70 years old; at least 2 teeth with dentin hypersensitivity in two different hemiarchs; dental elements with hypersensitivity without any pulp alteration; subjects not under any medication and non-pregnant women; subjects without any eating disorders or with very acidic diets. Further, exclusion criteria comprised subjects whose teeth with hypersensitivity featured extensive or unsatisfactory restorations, fractures, periodontal alterations, caries lesions, orthodontic brackets, endodontic treatments or pulp alterations; subjects who received desensitizing therapy during the last 3 months; subjects under analgesics/ anti-inflammatory drugs at the time of the study; subjects with non-carious cervical lesions more than $1 \mathrm{~mm}$ deep with dentinal cavitation featuring dentin hypersensitivity. Figure shows study design. Current researchers evaluated, during a 6-month period, twenty volunteers (17 females and 3 males), age bracket $20-63$ (mean age: $42.7 \pm 13.2$ years), who had a total of 152 teeth affected by dentin hypersensitivity. 


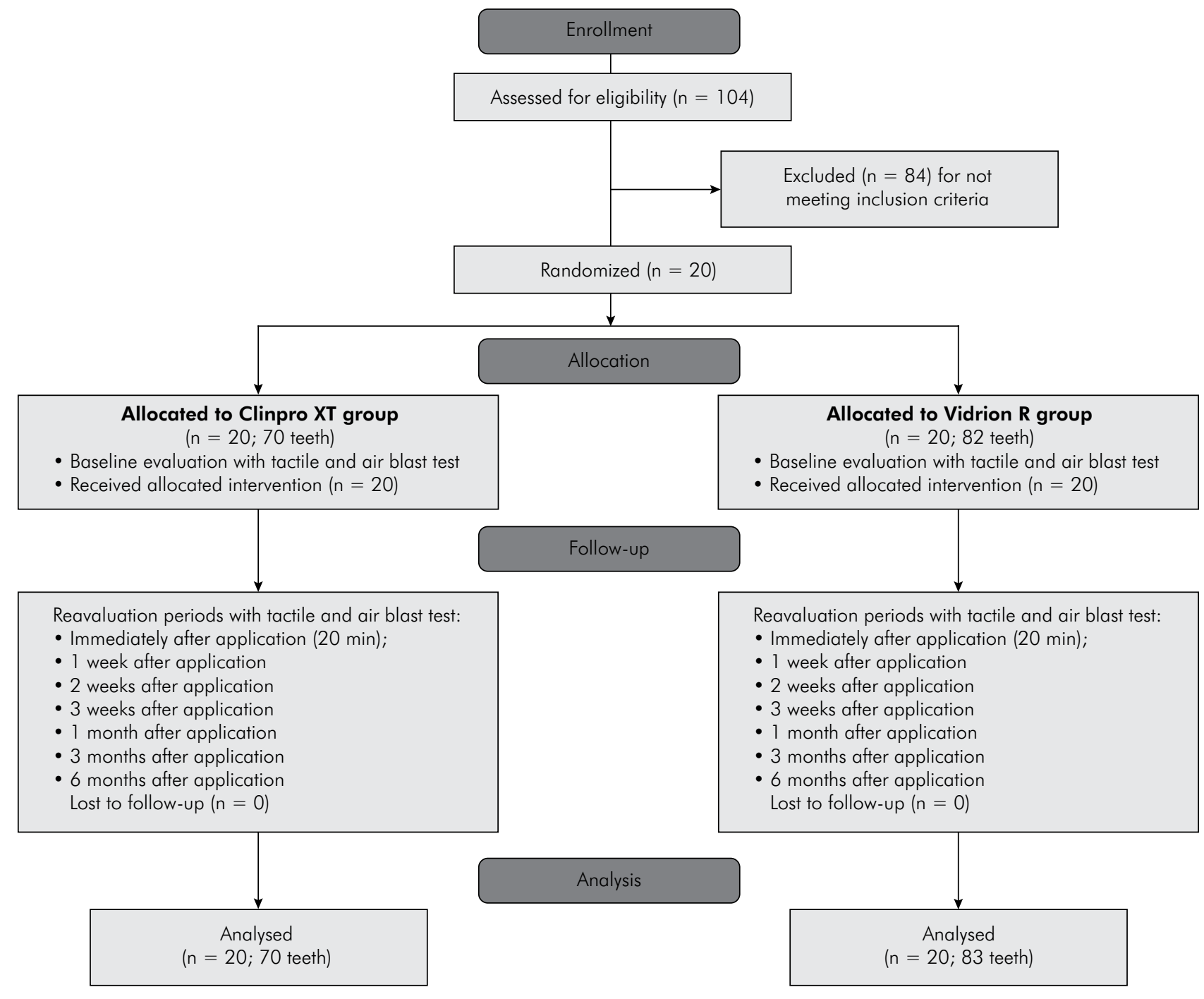

Figure. Flowchart of the study design.

\section{Application of glass ionomer cements}

A paired (split-mouth) study design was performed with sagittal midline as reference. The teeth from each side of the mouth (left or right) were randomly allocated (by chance, in a sealed envelope). Each material was applied to all the affected teeth from the same side of the mouth, employing isolation with cotton rolls, vacuum aspirator and mouth retractor.

The sensitive tooth surface was cleaned and rinsed with water for the application of Clinpro ${ }^{\mathrm{TM}} \mathrm{XT}$ (3M ESPE, Minnesota, USA, Batch number: 12248). Pooled water was removed and the tooth was left slightly moist. Liquid and paste of the material were mixed (10-15 s) and a thin layer ( $0.5 \mathrm{~mm}$ or less) was applied to the sensitive tooth surface and light-cured for 20 seconds. Ledges or overhangs were trimmed. Further, the sensitive tooth surface was cleaned and pooled water was removed for the application of Vidrion R (SS White, Gloucester, UK, Batch number: 59631). Liquid and powder were mixed (45-60 s) and a thin layer ( $0.5 \mathrm{~mm}$ or less) was applied to the sensitive tooth surface. After $15 \mathrm{~min}$, any ledges or overhangs were trimmed. Clinpro ${ }^{\mathrm{TM}}$ XT (3M ESPE, Minnesota, USA) and Vidrion R (SS White, Gloucester, UK) were used for at least two teeth of different hemiarchs in each subject who was blinded to treatment conditions.

All subjects received verbal and written information that no other oral hygiene product with a desensitizing 
effect should be used. In addition, they received professional instructions on adequate oral hygiene. All subjects were also provided with a soft toothbrush (Oral-B Pro-Health Toothbrush ${ }^{\mathrm{TM}}$, Procter \& Gamble Co., Ohio, USA) and a dentifrice with no desensitizing effect (Oral-B Pro-Health ${ }^{\mathrm{TM}}$, Procter \& Gamble Co., Ohio, USA; with 1450ppm fluoride) after the application of dental materials.

\section{Measurement protocol}

The sensitivity/pain response was assessed using the numerical (range 0-10) Visual Analogue Scale (VAS), with 0 indicating 'no pain' and 10 indicating 'intolerably severe pain'. Pre-treatment sensitivity (baseline) was evaluated by one researcher (WLOR), with the following test stimuli:

a. Tactile test (mechanical method): A sharp dental explorer (17/23) was passed lightly across the affected area, perpendicular to the long axis of the tooth. The test was repeated three times before the score was recorded.

b. Air blast test: An air blast from a dental syringe at 60-pound/inches ${ }^{2}$ pressure was directed on the tooth for 3 seconds from a distance of approximately $5 \mathrm{~mm}$.

After randomization, the treatment was applied by another researcher (MMM). Further, the first researcher (WLOR) evaluated the post treatment VAS response by applying test stimuli immediately after treatment (approximately 20 minutes) and at 7 days, 14 days, 21 days, 1 month, 3 months and 6 months. So that the standardization of the distance between the air blast and the sensitive tooth surface could be ensured, the air blast was applied adjacent to a periodontal probe from a $1 \mathrm{~cm}$-distance, without touching the tooth surface. Neighboring teeth were isolated with cotton rolls and suction devices to prevent false responses.

\section{Statistical analyses}

All VAS scores from each test stimuli were appropriately tabulated. Data were analyzed taking into consideration the teeth from the respective patient using the time periods evaluated in a repeated measured pattern. Friedman's test detected significant differences for scores of tactile test or air blast test, for each material and when significant; Tukey's post-hoc test was applied for pairwise comparisons $(\alpha=5 \%)$, using SigmaPlot 12.0 software (Systat Software Inc., Chicago IL, USA).

Mann-Whitney analysis independently compared the treatments according to specific VAS method (tactile test or air blast test). Data underwent Kruskal-Wallis (patient as unit of analysis) analysis followed by Tukey's test for comparison between materials and focused for each evaluation period investigated. Additionally, the correlation between the evaluation methods (tactile and air blast test) was determined by Spearman's rank correlations.

\section{Results}

All 20 subjects featuring 152 teeth with dentin hypersensitivity at the buccal surfaces completed the 6-month study period. Most teeth with DH were maxillary or mandibular premolars $(42.8 \%)$, followed by molars (20.4\%), incisor (19.1\%) and canine (17.8\%). In this split-mouth study design, $46 \%(n=70)$ of teeth with $\mathrm{DH}$ were randomly selected to receive Clinpro XT, while 54\% $(\mathrm{n}=82)$ received Vidrion $\mathrm{R}$. The range of teeth evaluated by subject was of 2 to 10 . No complications, such as adverse pulp effects, were observed throughout the study.

Subjects' response to tactile and air blast stimuli throughout the study is shown in Table. Regardless of the material, overall VAS scores revealed that medians in the air blast test were statistically higher $(p<0.001)$ than those in the tactile test.

A statistically significant difference was detected by Friedman's analysis ( $p<0.001)$ after six months for tactile and air blast tests (VAS scores). When compared to baseline, all evaluation times under analysis provided significantly lower means and indicated a reduction of pain sensitivity scores $(p<0.05)$. No significant differences in the reduction of painful symptoms were detected when treatments with Clinpro XT and Vidrion R were compared at same evaluation time on the response to tactile and air blast test stimuli. Spearman's rank correlation was applied to the evaluation methods (tactile and air blast test) and revealed a significant but moderate correlation $\left(r^{2}=0.53 ; p<0.001\right)$. 
Table. VAS medians (first and third quartile) for Clinpro XT and Vidrion R in tactile and air blast tests.

\begin{tabular}{|c|c|c|c|c|}
\hline \multirow{2}{*}{ Evaluation period } & \multicolumn{2}{|c|}{ Tactile test } & \multicolumn{2}{|c|}{ Air blast test } \\
\hline & Clinpro XT & Vidrion $\mathrm{R}$ & Clinpro XT & Vidrion R \\
\hline Baseline & $1.70(0.55-5.75)^{*}$ & $2.60(0.90-5.70)^{*}$ & $5.10(1.60-7.55)^{*}$ & $6.00(1.10-8.40)^{*}$ \\
\hline 20 minutes & $0.60(0.05-2.25)$ & $0.20(0.00-1.55)$ & $1.40(0.30-5.25)$ & $1.80(0.25-4.65)$ \\
\hline 7 days & $0.50(0.00-4.25)$ & $0.10(0.00-0.70)$ & $0.80(0.10-3.55)$ & $1.00(0.10-4.00)$ \\
\hline 15 days & $0.20(0.00-1.60)$ & $0.20(0.00-0.75)$ & $0.70(0.10-3.65)$ & $1.00(0.20-3.10)$ \\
\hline 21 days & $0.20(0.00-1.15)$ & $0.10(0.00-1.00)$ & $0.80(0.10-2.60)$ & $0.50(0.10-2.30)$ \\
\hline 30 days & $0.20(0.00-1.50)$ & $0.10(0.00-0.50)$ & $0.90(0.00-2.95)$ & $0.80(0.00-2.25)$ \\
\hline 90 days & $0.10(0.00-1.05)$ & $0.10(0.00-0.45)$ & $0.60(0.00-2.75)$ & $0.50(0.10-2.00)$ \\
\hline 180 days & $0.20(0.00-0.40)$ & $0.30(0.00-0.50)$ & $0.40(0.00-1.80)$ & $0.20(0.05-1.60)$ \\
\hline
\end{tabular}

*Statistically different from other groups $(p<0.05)$.

\section{Discussion}

Several treatment options are available for managing dentin hypersensitivity, and the rapid reduction of $\mathrm{DH}$ and long-term duration of these desensitizing effects are critical. Previous studies reported that the effects of many desensitizing agents were not permanent because tubule occlusion failed to be sustained (acidic conditions would remove dentin occlusion or tooth brushing would remove the restoration). ${ }^{12}$ Resinmodified GICs showed satisfactory long-term results in class $\mathrm{V}$ restorations, with a survival rate of $78.6 \%$ over a 5-year follow-up. ${ }^{2}$ Thus, the material may be employed as a long-term desensitizing material to control dentin hypersensitivity. To the best of our knowledge, this is the first randomized clinical trial that evaluated the long-term clinical outcome of a desensitizing treatment with the resin-modified glass ionomer cement (Clinpro XT) compared to a conventional GIC (Vidrion R). Current results demonstrated that both treatments were able to immediately decrease pain symptoms after application and maintain this effect during a 6-month follow-up. However, our initial hypothesis that the Clinpro XT would exhibit superior long-term effects when compared with Vidrion R was not proven.

Glass ionomer cements may form chemical bonds with enamel and dentin, release fluoride, promote a good biological response (biocompatibility) and have a coefficient of thermal expansion close to that of tooth structures. ${ }^{21}$ In current study, both GIC treatments decreased pain symptoms. In fact, the material binds with enamel and dentin via ionic and polar bonds, and close molecular contact facilitates the exchange of fluoride ions with hydroxyl ions at the surrounding dental apatite. The setting chemistry of glass ionomer cement is an acidbase reaction between polyalkenoic acid and fluoridecontaining aluminosilicate glass. Although fluoride ions may be released during the acid-base reaction, ${ }^{11}$ the ability of these materials to cover exposed dentin is their main mechanism that causes $\mathrm{DH}$ reduction.

Clinpro XT, a resin-modified glass ionomer cement based on methacrylate-modified polyalkenoic acid technology, may be applied in a thin layer to dental substrate (approximately $0.5 \mathrm{~mm}$ ). ${ }^{6}$ A recent randomized clinical trial was conducted with 31 patients and 119 teeth, evaluating the effect of Clinpro XT in reducing dentin hypersensitivity for up to 4 weeks. ${ }^{14}$ The study compared resin-modified GIC with Gluma Desensitizer (Heraeus Kulzer Inc., Wehrheim, Germany) and a placebo control, evaluating pain symptomatology with tactile and air blast test. Clinpro XT proved to be more effective than other groups at reducing DH immediately and for up to 4 weeks. Current study demonstrated that Clinpro $\mathrm{XT}$ was equivalent to conventional glass ionomer cement up to a 6-month post-treatment period. Other studies demonstrated that resin-modified GICs may have a higher remineralizing capacity. Fluoride catalyzes the diffusion of calcium and phosphate into the tooth surface and remineralizes dental substrates. Further, acid-base reaction enhances the leaching of fluoride ions to form a polysalt matrix, and resin-modified glass ionomer cements, such as Clinpro XT, revealed a more controlled and sustained release of remineralization agents over a 6-week time period. ${ }^{14}$ This effect suggests an improvement 
in long-term $\mathrm{DH}$ treatment. However, a significant decrease was detected after an initial period of fluoride release. ${ }^{13,22}$ An in vitro study with Clinpro $\mathrm{XT}^{(23)}$ showed that resin-based desensitizers did not release sufficient levels of fluoride ions to produce a clinically remarkable impact. Further studies are necessary to evaluate whether fluoride release by these materials has a beneficial effect on relieving $\mathrm{DH}$. As observed in current clinical trial, the effects of Clinpro XT were equivalent to the conventional glass ionomer cement Vidrion R, probably due to the physical occlusion of dentinal tubules.

Although dentin hypersensitivity may affect any tooth, it frequently involves canines and premolars. ${ }^{11}$ Contrastingly to other studies, premolars and molars were the main teeth affected in current clinical trial. The researchers included any teeth with $\mathrm{DH}$ to obtain the best overall results for both treatments evaluated, regardless of type of teeth. DH-affected teeth tend to vary among studies and populations. In fact, the literature has registered different distribution patterns. ${ }^{4,24,25}$

In previous clinical trials evaluating painful sensitivity in subjects, the use of more than one stimulus to evaluate this subjective condition has been recommended. ${ }^{3,426,27}$ In current analysis, hypersensitive teeth were evaluated using a tactile test with a sharp dental explorer and an evaporative stimulus via an air blast from a dental air syringe. These methods were previously effective in the diagnosis and evaluation of $\mathrm{DH} .{ }^{28}$ Pain is a rather subjective item and the assessment of the therapeutic effect of a $\mathrm{DH}$ treatment may be difficult., ${ }^{42}$ However, the Visual AnalogueScale (VAS) is widely used and generally accepted for the assessment of pain.,29 Despite the high number of subjects screened $(n=104), 20$ subjects were included based on the study design (split-mouth) and eligibility criteria.

Additionally, a placebo effect may influence the results in clinical trials that evaluate subjective items such as pain symptomatology. ${ }^{30}$ The interpretation of the benefits by using GIC should take into account the placebo effect, since a change in pain perception due to treatment may occur and has been well documented in DH studies. ${ }^{2}$ Although current study did not compare Clinpro XT with a placebo control, another recent randomized clinical trial showed that resin-modified GIC was more effective than the placebo in reducing DH for up to 4 weeks. ${ }^{2,29,31,32,33}$ Further, a split-mouth study design was adopted to eliminate the effects of individual conceptions of pain, pain perception, oral hygiene habits, dietary habits and psychosomatic factors. ${ }^{14}$

Although the ultimate test of any treatment option is how well it works in clinical practice, the randomized, controlled and double-blind clinical trial is the gold standard for determining efficacy. ${ }^{34}$ This is also the first randomized clinical trial that evaluated Clinpro XT and compared the resin-modified GIC with a conventional GIC. Whereas a recent study showed that Clinpro XT reduced $\mathrm{DH}$ in up to 4 and 12 weeks, current results demonstrated that this effect may be long-term.

It is important to emphasize the clinical advantages of Clinpro XT when compared with conventional GICs. Modified GIC is easy to use; it does not require a rubber dam and it is moisture-tolerant, which facilitates its clinical use in regions that requires strict moisture control, such as the cervical dental region. ${ }^{4,28}$ Besides, conventional GIC, such as Vidrion R, is indicated for non-carious cervical lesions, and they are more critical to apply and to reach the $0.5 \mathrm{~mm}$ layer. In addition, resin-modified GICs improved aesthetic properties and thus greater patient's acceptance over conventional GICs. ${ }^{14,35}$ However, modified GIC is more expensive and costs about six times more than conventional GIC. Further, other factors such as retention rate, stain, gingival response and plaque accumulation may also be relevant in the treatment of dentin hypersensitivity and must be taken into consideration by dentists. Since the two materials showed similar results for reducing dentin hypersensitivity over time, the dentist must evaluate which material would be most suitable for each clinical case. Future reevaluations will be conducted to verify the effectiveness of these treatments over a 6-month time period.

\section{Conclusion}

Both conventional and resin-modified glass ionomer cements showed similar results in pain reduction in a 6-month follow-up period and the two products proved to provide satisfactory results for the clinical management of dentin hypersensitivity. 


\section{References}

1. Brännström M. The hydrodynamics of the dental tubule and pulp fluid: its significance in relation to dentinal sensitivity. Annu Meet Am Inst Oral Biol. 1966;23:219.

2. Rosa WLO, Lund RG, Piva E, Silva AF. The effectiveness of current dentin desensitizing agents used to treat dental hypersensitivity: a systematic review. Quintessence Int. 2013;44(7):535-46. doi:10.3290/j.qi.a29610

3. Lund RG, Silva AF, Piva E, Rosa WL, Heckmann SS, Demarco FF. Clinical evaluation of two desensitizing treatments in southern Brazil: a 3-month follow-up. Acta odont Scand. 2013;71(6):1469-74. doi:10.3109/00016357.2013.770919

4. Orchardson R, Gillam DG. Managing dentin hypersensitivity. J Am Dent Assoc. 2006;137(7):990-8. doi:10.14219/jada.archive.2006.0321

5. Rees JS, Addy M. A cross-sectional study of dentine hypersensitivity. J Clin Periodontol. 2002;29(11):997-1003. doi:10.1034/j.1600-051X.2002.291104.x

6. Neelakantan P, John S, Anand S, Sureshbabu N, Subbarao C. Fluoride release from a new glass-ionomer cement. Oper Dent. 2011;36(1):80-5. doi:10.2341/10-219-LR

7. Franco EB, Benetti AR, Ishikiriama SK, Santiago SL, Lauris JR, Jorge MF et al. 5-year clinical performance of resin composite versus resin modified glass ionomer restorative system in non-carious cervical lesions. Oper Dent. 2006;31(4):403-8. doi:10.2341/05-87

8. Yan Z, Sidhu SK, Mahmoud GA, Carrick TE, McCabe JF. Effects of temperature on the fluoride release and recharging ability of glass ionomers. Oper Dent. 2007;32(2):138-43. doi:10.2341/06-36

9. Ozgünaltay G, Onen A. Three-year clinical evaluation of a resin modified glass-ionomer cement and a composite resin in non-carious class V lesions. J Oral Rehabil. 2002;29(11):1037-41. doi:10.1046/j.1365-2842.2002.00995.x

10. Naorungroj S, Wei HH, Arnold RR, Swift EJ Jr, Walter R. Antibacterial surface properties of fluoride-containing resin-based sealants. J Dent. 2010;38(5):387-91. doi:10.1016/j.jdent.2010.01.005

11. Sohn S, Yi K, Son HH, Chang J. Caries-preventive activity of fluoride-containing resin-based desensitizers. Oper Dent. 2012;37(3):306-15. doi:10.2341/11-007-L

12. Tantbirojn D, Poolthong S, Leevailoj C, Srisawasdi S, Hodges JS, Randall RC. Clinical evaluation of a resin-modified glass-ionomer liner for cervical dentin hypersensitivity treatment. Am J Dent. 2006;19(1):56-60.

13. Zhou SL, Zhou J, Watanabe S, Watanabe K, Wen LY, Xuan $K$. In vitro study of the effects of fluoride-releasing dental materials on remineralization in an enamel erosion model. J Dent. 2012;40(3):255-63. doi:10.1016/j.jdent.2011.12.016

14. Ding YJ, Yao H, Wang GH, Song H. A randomized double-blind placebo-controlled study of the efficacy of
Clinpro XT varnish and Gluma dentin desensitizer on dentin hypersensitivity. Am J Dent. 2014;27(2):79-83.

15. Beun S, Bailly C, Devaux J, Leloup G. Physical, mechanical and rheological characterization of resin-based pit and fissure sealants compared to flowable resin composites. Dent Mater. 2012;28(4):349-59. doi:10.1016/j.dental.2011.11.001

16. Nicholson JW, Czarnecka B. The biocompatibility of resin-modified glass-ionomer cements for dentistry. Dent Mater. 2008;24(12):1702-8. doi:10.1016/j.dental.2008.04.005

17. Kovarik RE, Haubenreich JE, Gore D. Glass ionomer cements: a review of composition, chemistry, and biocompatibility as a dental and medical implant material. J Long Term Eff Med Implants. 2005;15(6):655-71. doi:10.1615/JLongTermEffMedImplants.v15.i6.80

18. Yilmaz HG, Kurtulmus-Yilmaz S, Cengiz E, Bayindir H, Aykac Y. Clinical evaluation of Er,Cr:YSGG and GaAlAs laser therapy for treating dentine hypersensitivity: a randomized controlled clinical trial. J Dent. 2011;39(3):249-54. doi:10.1016/j.jdent.2011.01.003

19. Rosa WLO, Silva AF, Oliveira AS, Lund RG, Leites ACBR, Piva E. Technological monitoring of dentin desensitizing agents. RFO UPF. 2014;19(1):107-14.

20. Moher D, Hopewell S, Schulz KF, Montori V, Gøtzsche PC, Devereaux PJ et al. CONSORT 2010 explanation and elaboration: updated guidelines for reporting parallel group randomised trials. BMJ. 2010;340:c869. doi:10.1136/bmj.c869

21. Stewardson D, Creanor S, Thornley P, Bigg T, Bromage $\mathrm{C}$, Browne A et al. The survival of Class $\mathrm{V}$ restorations in general dental practice: part 3, five-year survival. Br Dent J. 2012;212(9):E14. doi:10.1038/sj.bdj.2012.367

22. Chan WD, Yang L, Wan W, Rizkalla AS. Fluoride release from dental cements and composites: a mechanistic study. Dent Mater. 2006;22(4):366-73. doi:10.1016/j.dental.2005.04.028

23. Hara AT, Queiroz CS, Freitas PM, Giannini M, Serra MC, Cury JA. Fluoride release and secondary caries inhibition by adhesive systems on root dentine. Eur J Oral Sci. 2005;113(3):245-50. doi:10.1111/j.1600-0722.2005.00214.x

24. Magno MB, Nascimento GC, Da Penha NK, Pessoa OF, Loretto SC, Maia LC. Difference in effectiveness between strontium acetate and arginine-based toothpastes to relieve dentin hypersensitivity: a systematic review. Am J Dent. 2015;28(1):40-4.

25. Sharif MO, Iram S, Brunton PA. Effectiveness of arginine-containing toothpastes in treating dentine hypersensitivity: a systematic review. J Dent. 2013;41(6):483-92. doi:10.1016/j.jdent.2013.01.009

26. He T, Barker ML, Biesbrock AR, Miner M, Qaqish J, Sharma N. A clinical study to assess the effect of a stabilized stannous fluoride dentifrice on hypersensitivity relative to a marketed sodium fluoride/triclosan control. J Clin Dent. 2014;25(2):13-8. 
Evaluation of dentin hypersensitivity treatment with glass ionomer cements: a randomized clinical trial

27. Rees JS, Jin LJ, Lam S, Kudanowska I, Vowles R. The prevalence of dentine hypersensitivity in a hospital clinic population in Hong Kong. J Dent. 2003;31(7):453-61. doi:10.1016/S0300-5712(03)00092-7

28. Holland GR, Narhi MN, Addy M, Gangarosa L, Orchardson R. Guidelines for the design and conduct of clinical trials on dentine hypersensitivity. J Clin Periodontol. 1997;24(11):808-13. doi:10.1111/j.1600-051X.1997.tb01194.x

29. Addy M, West NX, Barlow A, Smith S. Dentine hypersensitivity: is there both stimulus and placebo responses in clinical trials? Int J Dent Hygiene. 2007; 5(1):53-59. doi:10.1111/j.1601-5037.2007.00228.x

30. Lier BB, Rösing CK, Aass AM, Gjermo P. Treatment of dentin hypersensitivity by Nd:YAG laser. J Clin Periodontol. 2002;29(6):501-6. doi:10.1034/j.1600-051X.2002.290605.x

31. Cunha-Cruz J, Stout JR, Heaton LJ, Wataha JC, Northwest P. Dentin hypersensitivity and oxalates: a systematic review. J Dent Res. 2011;90(3):304-10. doi:10.1177/0022034510389179

32. He S, Wang Y, Li X, Hu D. Effectiveness of laser therapy and topical desensitising agents in treating dentine hypersensitivity: a systematic review. J Oral Rehabil. 2011;38(5):348-58. doi:10.1111/j.1365-2842.2010.02193.x

33. Poulsen S, Errboe M, Lescay Mevil Y, Glenny AM. Potassium containing toothpastes for dentine hypersensitivity. Cochrane Database Syst Rev. 2006(3):CD001476. doi:10.1002/14651858.CD001476.pub2

34. Drebenstedt S, Zapf A, Rödig T, Mausberg R, Ziebolz D. Efficacy of two different CHX-containing desensitizers: a controlled double-blind study. Oper Dent. 2012;37(2):161-71. doi:10.2341/10-231-C

35. Francisconi LF, Scaffa PM, de Barros VR, Coutinho M, Francisconi PA. Glass ionomer cements and their role in the restoration of non-carious cervical lesions. J Appl Oral Sc. 2009;17(5):364-9. doi:10.1590/S1678-77572009000500003 